\title{
LA CLASIFICACIÓN DE LOS MÉTODOS DE ENSEÑANZA EN EDUCACIÓN SUPERIOR
}

\author{
Jesús Alcoba González \\ Lasalle Campus Madrid
}

\begin{abstract}
RESUMEN: El presente artículo plantea algunas de las dificultades terminológicas y taxonómicas en el estudio de los métodos de enseñanza. Como propuesta para superar estas dificultades se aportan definiciones de algunos conceptos clave en este campo y se describe la elaboración de un listado de métodos de enseñanza con sus definiciones, instrumento que juzgamos imprescindible de cara al desarrollo de competencias en el modelo planteado por el Espacio Europeo de Educación Superior. Esta relación de métodos se ha realizado a partir de veintidós fuentes documentales y un panel de nueve expertos, y persigue los siguientes objetivos: proporcionar a los docentes una herramienta para el diseño de guías académicas, ayudar a los procesos de diseño de acciones formativas para el profesorado y servir a la investigación en este campo.
\end{abstract}

PALABRAS CLAVE: Competencia, educación superior, método de enseñanza, metodología docente.

\section{ON CLASSIFYING TEACHING METHODS IN HIGHER EDUCATION}

ABSTRACT: This paper poses some of the terminological and taxonomic difficulties involved in the research of teaching methods. As an approach to overcome those difficulties we contribute with definitions of key concepts in this area and we describe the construction of a list of teaching methods with their definitions, a tool we deem essential for the development of competences in the model designed by the European Higher Education Area. The list has been devised from twenty-two documentary sources and a nine-expert panel and intends to provide teachers with a tool for designing academic guides, help with teacher training design processes and contribute to this research field.

KEYWORDS: Competence, higher education, teaching method, teaching methodology. 


\section{INTRODUCCIÓN}

La metodología es uno de los elementos imprescindibles en la elaboración de unidades didácticas desde que fuera incluida en el modelo de Glaser (Ferrández y Sarramona, 1975). Sin embargo, y pese a lo mucho que se ha escrito sobre ella, aún no es posible encontrar una clasificación de métodos de enseñanza práctica y generalizada que facilite su uso por parte de los docentes. Se trata de un problema importante, pues por un lado el profesorado puede notar una falta de opciones metodológicas de cara al desarrollo de competencias, y por otro se dificultan las acciones de formación del mismo. Adicionalmente, la construcción de instrumentos de recogida de información para investigar en este campo se vuelve también compleja.

Como aproximación a estos problemas el presente trabajo propone una delimitación conceptual y muestra el proceso de construcción de un listado de métodos de enseñanza utilizado en una investigación sobre la estrategia docente de las instituciones de Educación Superior (Alcoba, 2010) así como una versión revisada de dicha relación.

Es común en la literatura científica sobre métodos de enseñanza encontrar listados de métodos que cada autor enumera según su experiencia y conocimiento, siendo ciertamente infrecuente que unos autores se apoyen en la obra de otros. Esto posiblemente es la causa de los diversos problemas que surgen en la investigación en este campo, como son los que mencionamos a continuación.

Una de las primeras dificultades que surge tras el análisis de distintos autores es la gran dispersión que existe en la enumeración de métodos. Así por ejemplo, la relación del Ministerio de Educación y Ciencia (MEC, 2006) incluye solo tres métodos, pero la de Navaridas (2004) incorpora veinticinco, y la de Bourner (1997) más de cincuenta.

Otra dificultad es que los autores no utilizan los mismos términos para referirse a métodos que parecen similares. Así por ejemplo Amat y De Miguel (Amat, 2002; De Miguel, 2006) usan el término lección magistral, mientras que la Universidad Rovira i Virgili se refiere a este método como sesión magistral (URV, 2006), y Zabalza (2003) citando a Tejada (2001) utiliza método magistral. Jares cita un método conocido como dinámica de clarificación de valores (Jares, 2002), pero para el Community College de Honolulu este método se llama ejercicio de clarificación de valores (Honolulu Community College, s.f.). Otro ejemplo lo encontramos en Navaridas y De Miguel, que hablan de estudio de casos (De Miguel, 2006; Navaridas, 2004), mientras que López Camps se refiere a ello como método del caso (López Camps, 2005).

Por otro lado, diremos que no siempre es fácil saber si los autores usan diversos términos porque se refieren a métodos distintos, debido a que no siempre definen los términos que usan. En siete de las fuentes que hemos consultado (aproximadamente una de cada tres) ocurre este fenómeno. Esta cuestión se complica porque las definiciones utilizadas por algunos autores para determinados métodos son similares a las que otros mencionan para métodos diferentes, o porque algunos métodos parecen englobar a otros. Por citar un ejemplo, Kolb definió el aprendizaje como el proceso a través del cual se crea conocimiento a través de la transformación de la 
experiencia (Kolb, 1984). Desde esta definición, y aunque en algunas fuentes el aprendizaje experiencial aparezca como un método de enseñanza (Bourner, 1997; BusinessWeek, 2006; Monterrey, 2006), es plausible asimismo considerarlo como un enfoque que engloba a todos aquellos métodos en los que el alumno entra directamente en contacto con la realidad para aprender o descubrir algo, como por ejemplo ocurre en las prácticas de laboratorio.

Otra dificultad que encontramos es que algunos autores unen unos métodos a otros como muestra de su similitud, pero estos parecidos no son compartidos por otros autores. Así por ejemplo, Jares habla de juegos y ejercicios de simulación (Jares, 2002) y Fernández March de simulación y juego (Fernández March, 2006). Sin embargo, Navaridas y el TEC de Monterrey solo hablan de simulación (Monterrey, 2006; Navaridas, 2004). Por otro lado, Gairín escribe simulación/role playing (Gairín, 2005), pero el juego de roles aparece aislado en otros autores, como por ejemplo en Amat y en López Camps (Amat, 2002; López Camps, 2005). Para este último autor, finalmente, el método del caso forma parte de los juegos y simulaciones, mientras que en otros autores encontramos ambos métodos por separado, como por ejemplo en Domínguez et. al o en Navaridas (Domínguez, Amador, Hermosilla y Lozano, 2007; Navaridas, 2004).

Por último, si nos fijamos en los objetivos que pretenden los diversos métodos, tampoco parece haber acuerdo entre los distintos autores. Por ejemplo Amat refiere que los métodos que fomentan la creatividad son la tutoría, el Phillips 66 y la tormenta de ideas (Amat, 2002), y sin embargo para Navaridas la tutoría es uno de los métodos orientados al cambio de conocimientos, concretamente una de las estrategias individualizadoras. Para él, los métodos que fomentan la creatividad son el torbellino de ideas, la interrogación didáctica, las técnicas audiovisuales y el método del incidente (Navaridas, 2004). Pero Bourner, sin embargo, amplía el listado de métodos que sirven para que el estudiante genere ideas a diez, entre los que resulta llamativo encontrar las técnicas de relajación (Bourner, 1997).

\section{DELIMITACIÓN CONCEPTUAL}

La ausencia de terminología unificada en el campo de los métodos de enseñanza es un hecho constatado, y por eso es común en este terreno que los autores comiencen por definir el significado que para ellos tienen los términos que usan (Fernández Pérez, 1994, p. 584).

En este trabajo utilizamos la expresión método de enseñanza para referirnos a la unidad básica de la metodología. En la revisión de listados de métodos de enseñanza que realizamos en este trabajo, nueve autores utilizaron este término (Sáenz, 1994; Bourner, 1997; Davis, Misra y Van Auken, 2000; Gil, Álvarez, García y Romero, 2004; De Miguel, 2006; Fernández March, 2006; Petty, 2006; BusinessWeek, 2008; Honolulu Community College, s.f.).

Los otros términos utilizados fueron: métodos y técnicas de formación (Domínguez et al., 2007), métodos didácticos (EuroFM, 2007; Zabalza, 2003), técnicas docentes (Fernández Serrat, 2007), metodologías educativas (MEC, 2006), técnicas didácticas (Monterrey, 2006), estrategias de enseñanza-aprendizaje (Andreu, 2005), estrategias metodológicas (Gairín, 2005), métodos formativos (López Camps, 2005), 
estrategias didácticas (Navaridas, 2004), métodos pedagógicos (Amat, 2002), actividades didácticas (Jares, 2002), y metodologías docentes (URV, 2006).

Desde nuestra perspectiva un método de enseñanza es el conjunto de técnicas y actividades que un profesor utiliza con el fin de lograr uno o varios objetivos educativos, que tiene sentido como un todo y que responde a una denominación conocida y compartida por la comunidad científica.

Resaltamos que método es un término de índole más genérica que actividad o técnica, pero más concreta que modelo, y destacamos la relación que existe entre el método y los fines que pretende, que bajo nuestro punto de vista no son otros que las finalidades educativas en sus diversos niveles de concreción. Ahora bien, para que un método sea tal ( $\mathrm{y}$ no una técnica o una actividad), debe tener sentido como un todo, es decir, debe poder diferenciarse claramente cuándo un profesor está usando un método y cuándo otro. Así, en su ejecución, la clase magistral no debe ser lo mismo que el método del caso, ni la resolución de ejercicios y problemas deben ser lo mismo que las prácticas profesionales. Por último, un método de enseñanza debe tener un nombre reconocido y compartido por la comunidad científica. Un método no lo es hasta que se investiga y comunica sobre él. Un profesor puede diseñar un gran número de actividades, pero los métodos (y probablemente las técnicas) pertenecen a una escala de complejidad superior y para constituirse como tales debe haber cierto acuerdo en la comunidad científica sobre ellos.

Resulta de interés también clarificar el significado del término metodología. Según el Diccionario de la Real Academia, la metodología es la "ciencia del método", o bien el "conjunto de métodos que se siguen en una investigación científica o en una exposición doctrinal". (RAE, 2008). En la primera acepción nos estamos refiriendo por tanto a un conocimiento científico y en la segunda a un conjunto de métodos. Desde este punto de vista, metodología educativa es, en primer lugar, el área de la Ciencia que estudia los métodos de enseñanza. Y, en segundo lugar, la metodología es el conjunto de métodos utilizados por un docente.

La primera de las acepciones establece una relación entre ambos términos que desde nuestro enfoque resulta crucial, puesto que establece una conexión clara entre la disciplina, la metodología, y su objeto de estudio, que es el método (Martín-Molero, 1993, p. 52). Esta relación no existe si se escogen otros términos (la metodología no es el estudio de las técnicas ni de las estrategias, por ejemplo). Para nosotros por tanto la metodología es la relación de métodos de enseñanza que usa un profesor y su articulación en el aula y no, por ejemplo, la forma que este tiene de adjetivarlos. En otras palabras, lo que un profesor ha de consignar en el apartado de metodología dentro de una guía académica, como mínimo, es una enumeración de los métodos que usa y no, o al menos no solo, si esta es activa, si es participativa o si sigue o no los principios del aprendizaje significativo.

Bajo nuestra perspectiva las técnicas y las actividades son concreciones del conjunto de métodos que usan los docentes. Así, por citar un ejemplo dentro de las primeras, diremos que un ejercicio concreto de sensibilización puede ser considerado una técnica dentro del método general definido por la dinámica de grupos. De igual modo, hablar en público o la utilización de presentaciones con 
diapositivas pueden considerarse técnicas que forman parte del método conocido como lección magistral. Las actividades, por su parte, se sitúan en otro terreno de concreción, que incorpora el contenido de que se trate y que son contextualizadas dentro de una materia en particular: así por ejemplo, el visionado de una determinada película es una actividad, como también lo es la disección de un animal específico para su examen anatómico. El brainstorming y el Phillips 66 son técnicas de trabajo de grupo, es decir, técnicas dentro del método trabajo de grupo. Por el contrario, un brainstorming en particular orientado a lograr un resultado concreto dentro de un aula será una actividad. Recogemos en la tabla 1 algunos ejemplos clarificadores de la diferencia que existe entre estos tres términos.

Tabla 1. Ejemplos de métodos, técnicas y actividades

\begin{tabular}{|l|l|l|}
\hline MÉTODO & TÉCNICA & ACTIVIDAD \\
\hline Clase magistral. & $\begin{array}{l}\text { Hablar en público. } \\
\text { Presentación con diapositivas. }\end{array}$ & $\begin{array}{l}\text { Clase sobre "La influencia del lenguaje en } \\
\text { la construcción del pensamiento". }\end{array}$ \\
\hline Dinámica de grupos. & Ejercicio de sensibilización. & $\begin{array}{l}\text { Ejercicio de grupo sobre "La historia del } \\
\text { barquero". }\end{array}$ \\
\hline Prácticas de laboratorio. & Disección. & Disección de una rana. \\
\hline Audiovisual. & Videoforum. & $\begin{array}{l}\text { Videoforum sobre la película "Doce hom- } \\
\text { bres sin piedad". }\end{array}$ \\
\hline Trabajo de grupo. & Brainstorming. & $\begin{array}{l}\text { Brainstorming sobre las diferentes aplica- } \\
\text { ciones de la Psicología. }\end{array}$ \\
\hline
\end{tabular}

Fuente: (Alcoba, 2010).

\section{Construcción del Listado de métodos de ENSEÑANZA}

Tal y como hemos escrito líneas arriba, la investigación en métodos de enseñanza conlleva una serie de dificultades que hemos pretendido superar a través de las acciones que pasamos a enumerar.

Por un lado, buscamos contar con una cantidad abundante de fuentes. Como hemos mencionado, normalmente los autores basan sus listados en su propio conocimiento y experiencia. Sin embargo, nuestra investigación se basó en las veintidós fuentes que hemos mencionado más arriba, siendo el único inventario que conocemos basado en un número tan abundante de fuentes. Se trata de autores muy diversos, y en algunos casos sus trabajos son muy distantes en cuanto a su campo de estudio: algunos son reflexiones sobre la docencia universitaria, otros son trabajos de investigación y algunos más hablan acerca de la Educación en general. Decidimos incluirlos todos basándonos en el meta-análisis de Marzano (1998), en el que se recoge que el nivel académico de los estudiantes no influye de modo significativo en el efecto de un determinado método. Por tanto, a priori lo que funciona en un nivel educativo puede funcionar igualmente en otro. 
Por otro lado, y dada la dificultad terminológica existente, para las definiciones de cada uno de ellos partimos del único cuestionario de métodos de enseñanza que conocemos que incorpora un glosario, que es el de Navaridas (2004). En el caso de que la definición que aportaba el glosario a nuestro entender pudiera ser mejorada intentamos hacerlo, y en el caso de métodos no recogidos por el cuestionario recurrimos a fuentes externas.

Las fuentes analizadas arrojaron un total de 84 métodos de enseñanza. Pensábamos que era un número excesivamente elevado por su escasa practicidad, y para reducirlo consideramos únicamente aquellos métodos que habían sido citados al menos por dos autores, para garantizar un mínimo de acuerdo. Ahora bien, una de las dificultades consistió en definir cuándo había acuerdo entre los autores respecto a un método concreto. Pensemos que, como ya hemos dicho, los autores a veces utilizan términos distintos, y además no siempre definen los métodos en sus listados.

Las fuentes utilizadas nombraban individualmente un total de 247 métodos de enseñanza, y por tanto resultaría largo y complejo explicar en cada caso todas y cada una de las decisiones que fuimos tomando para determinar si había concurrencia en varios autores con respecto a un método. Pero sí citaremos algunos ejemplos: en primer lugar, consideramos que cuando había una importante similitud semántica en una parte del nombre del método, o en su significado, los autores se estaban refiriendo al mismo método. Por ejemplo asumimos que, en su esencia como método de enseñanza, lección es lo mismo que clase magistral. Por otro lado, si no encontramos diferencias significativas en la definición de dos métodos que nos parecían similares resolvimos agruparlos bajo una única denominación. Es el caso de debate, mesa redonda y coloquio. Un último ejemplo son los métodos que proceden de trabajos en inglés, en los que utilizamos un criterio de traducción amplio; así por ejemplo, tradujimos ex-cathedra teaching por clase magistral.

El primer listado que obtuvimos incorporaba treinta y nueve métodos de enseñanza y sus definiciones. Este trabajo fue sometido al juicio de nueve expertos, que formularon sus observaciones sobre los métodos y las definiciones recogidas. Así se construyó la primera versión del listado, inicialmente elaborado en el marco de una investigación sobre la estrategia docente de las instituciones de Educación Superior (Alcoba, 2010). Este listado sirvió en dicho estudio para lograr los siguientes objetivos:

- Utilizar un lenguaje común a la hora de referirse a los métodos de enseñanza.

- Servir de base para la elaboración de un cuestionario a través del cual se analizó el tejido metodológico de la institución en la cual tuvo lugar el estudio.

- Guiar los procesos de formación de profesores.

- Elaborar las guías académicas de las diferentes materias.

En la tabla 2 exponemos una versión revisada de aquel listado, que incorpora veinticinco métodos de enseñanza con sus definiciones. A pesar de que todas ellas han sido transformadas durante el proceso investigador, por rigor y fidelidad a los autores que las formularon en un inicio hemos resuelto incorporar las definiciones originales. Asimismo anotamos el número de fuentes que nombraron los distintos métodos de enseñanza, que es el criterio que hemos seguido para ordenarlos. Es 
decir, el primero de los métodos fue el más nombrado (20 de 22 fuentes), y el último el menos nombrado (2 de 22 fuentes).

Tabla 2. Listado de métodos de enseñanza

\begin{tabular}{|c|c|c|c|c|}
\hline & MÉTODO & $\begin{array}{c}\text { NÚMERO } \\
\text { DE } \\
\text { FUENTES }\end{array}$ & DEFINICIÓN ORIGINAL & DEFINICIÓN \\
\hline 1 & Clase magistral & 20 & $\begin{array}{l}\text { Método expositivo, generalmente } \\
\text { utilizado para facilitar información } \\
\text { actualizada y bien organizada } \\
\text { procedente de diversas fuentes y } \\
\text { de difícil acceso al estudiante } \\
\text { (Navaridas, 2004). }\end{array}$ & $\begin{array}{l}\text { Método cuya finalidad es la } \\
\text { exposición de un contenido } \\
\text { actualizado que ha sido } \\
\text { elaborado con finalidad } \\
\text { didáctica. }\end{array}$ \\
\hline 2 & Estudio de casos & 16 & $\begin{array}{l}\text { Un caso es la descripción de una } \\
\text { situación real o hipotética que } \\
\text { debe ser estudiada de forma } \\
\text { analítica y exhaustiva. Tiene por } \\
\text { objeto la capacitación práctica } \\
\text { para la solución de problemas } \\
\text { concretos (Navaridas, 2004). }\end{array}$ & $\begin{array}{l}\text { Un caso es el relato de una } \\
\text { situación que ha sido articulada } \\
\text { con el fin de lograr determinados } \\
\text { objetivos de aprendizaje. El caso } \\
\text { debe ser estudiado } \\
\text { exhaustivamente y plantea } \\
\text { problemas que los alumnos } \\
\text { deben resolver. }\end{array}$ \\
\hline 3 & Simulación & 13 & $\begin{array}{l}\text { Consiste en reproducir } \\
\text { acontecimientos o problemas } \\
\text { reales que no están accesibles a } \\
\text { los estudiantes y que, sin embargo, } \\
\text { su experimentación la consideramos } \\
\text { necesaria para su futuro profesional } \\
\text { (Navaridas, 2004). }\end{array}$ & $\begin{array}{l}\text { Representación de un } \\
\text { acontecimiento que } \\
\text { habitualmente no es accesible } \\
\text { para el estudiante con el fin de } \\
\text { estudiarlo en un entorno } \\
\text { simplificado y controlado. }\end{array}$ \\
\hline 4 & Proyectos & 12 & $\begin{array}{l}\text { Estrategia en la que el producto } \\
\text { del proceso de aprendizaje es un } \\
\text { proyecto o programa de } \\
\text { intervención profesional, en torno } \\
\text { al cual se articulan todas las } \\
\text { actividades formativas (Fernández } \\
\text { March, 2006). }\end{array}$ & $\begin{array}{l}\text { Trabajo cuya finalidad es un } \\
\text { producto concreto, } \\
\text { habitualmente condicionada por } \\
\text { unos requisitos de tiempo y } \\
\text { recursos, en la que la } \\
\text { planificación de tareas y la } \\
\text { resolución de incidencias cobran } \\
\text { especial importancia. }\end{array}$ \\
\hline 5 & Seminario & 10 & $\begin{array}{l}\text { Técnica de trabajo con pequeños } \\
\text { grupos de interés y nivel de } \\
\text { formación comunes. Permite } \\
\text { investigar con profundidad y de } \\
\text { forma colectiva un tema } \\
\text { especializado acudiendo a fuentes } \\
\text { originales de información } \\
\text { (Navaridas, 2004). }\end{array}$ & $\begin{array}{l}\text { Método en el que se articulan } \\
\text { uno o varios grupos de interés } \\
\text { con nivel de formación } \\
\text { habitualmente homogéneos. } \\
\text { Permite investigar con } \\
\text { profundidad y de forma colectiva } \\
\text { un tema especializado. }\end{array}$ \\
\hline 6 & Juego de roles & 9 & $\begin{array}{l}\text { Un grupo de estudiantes } \\
\text { representan una situación de la } \\
\text { realidad, para su posterior análisis } \\
\text { por el resto del grupo-clase. } \\
\text { Facilita la comprensión de un } \\
\text { problema, vivenciándolo en la } \\
\text { dramatización (Navaridas, 2004). }\end{array}$ & $\begin{array}{l}\text { Representación dramatizada de } \\
\text { una situación de la realidad en la } \\
\text { que los participantes representan } \\
\text { distintos papeles para su } \\
\text { ejercitación y estudio. }\end{array}$ \\
\hline
\end{tabular}




\begin{tabular}{|c|c|c|c|c|}
\hline & MÉTODO & $\begin{array}{c}\text { NÚMERO } \\
\text { DE } \\
\text { FUENTES }\end{array}$ & DEFINICIÓN ORIGINAL & DEFINICIÓN \\
\hline 7 & $\begin{array}{l}\text { Debate, mesa } \\
\text { redonda o } \\
\text { coloquio }\end{array}$ & 9 & $\begin{array}{l}\text { Confrontación de opiniones } \\
\text { distintas en una discusión } \\
\text { informal, bajo la dirección de un } \\
\text { moderador (Navaridas, 2004). }\end{array}$ & $\begin{array}{l}\text { Confrontación de opiniones en } \\
\text { torno a un tema bajo la dirección } \\
\text { de un moderador. Los } \\
\text { participantes pueden alinearse en } \\
\text { torno a dos o más posturas } \\
\text { dependiendo del formato. }\end{array}$ \\
\hline 8 & $\begin{array}{l}\text { Aprendizaje } \\
\text { basado en } \\
\text { problemas }\end{array}$ & 8 & $\begin{array}{l}\text { Estrategia en la que los } \\
\text { estudiantes aprenden en } \\
\text { pequeños grupos, partiendo de un } \\
\text { problema, a buscar la información } \\
\text { que necesitan para comprender el } \\
\text { problema y obtener una solución, } \\
\text { bajo la supervisión de un tutor } \\
\text { (Fernández March, 2006). }\end{array}$ & $\begin{array}{l}\text { Método en el que los estudiantes, } \\
\text { en grupo y partiendo de un } \\
\text { problema, determinan sus } \\
\text { objetivos de aprendizaje en } \\
\text { función de sus conocimientos y } \\
\text { buscan información para } \\
\text { comprender el problema y } \\
\text { obtener una solución con la } \\
\text { ayuda de un tutor. }\end{array}$ \\
\hline 9 & $\begin{array}{l}\text { Ejercicios y } \\
\text { problemas }\end{array}$ & 8 & $\begin{array}{l}\text { Metodología de entrenamiento } \\
\text { activa consistente en identificar } \\
\text { una situación conflictiva, definir } \\
\text { sus parámetros, formular y } \\
\text { desarrollar hipótesis y proponer } \\
\text { una solución o proponer } \\
\text { soluciones alternativas } \\
\text { (Navaridas, 2004). }\end{array}$ & $\begin{array}{l}\text { Tarea que consiste en solucionar } \\
\text { un problema o realizar una tarea } \\
\text { partiendo de los conocimientos } \\
\text { del estudiante y de una serie de } \\
\text { datos que se aportan en el } \\
\text { enunciado del problema. }\end{array}$ \\
\hline 10 & Tutorías & 7 & $\begin{array}{l}\text { Se asume la función tutorial como } \\
\text { un potente recurso metodológico } \\
\text { que el profesor puede utilizar para } \\
\text { individualizar la enseñanza y } \\
\text { ajustarla a las características } \\
\text { personales de cada estudiante } \\
\text { (asesorándole sobre la forma de } \\
\text { estudiar la asignatura, facilitándole } \\
\text { fuentes bibliográficas y } \\
\text { documentales concretas para la } \\
\text { resolución de un problema de } \\
\text { aprendizaje, etc.) (Navaridas, } \\
\text { 2004). }\end{array}$ & $\begin{array}{l}\text { Método cuyo fin es } \\
\text { individualizar la enseñanza y } \\
\text { ajustarla a las características de } \\
\text { cada estudiante asesorándole } \\
\text { sobre la forma de estudiar la } \\
\text { asignatura, facilitándole fuentes } \\
\text { bibliográficas, ayudándole en la } \\
\text { resolución de un problema o } \\
\text { guiándole en la realización de un } \\
\text { proyecto o investigación. }\end{array}$ \\
\hline 11 & Brainstorming & 6 & $\begin{array}{l}\text { Esta técnica tiene como objetivo } \\
\text { básico la producción de nuevas } \\
\text { ideas para su posterior reflexión. } \\
\text { Cada estudiante va diciendo lo } \\
\text { que se le ocurre sobre un tema } \\
\text { específico, dejando la crítica de } \\
\text { lado en un primer momento } \\
\text { (Navaridas, 2004). }\end{array}$ & $\begin{array}{l}\text { Con el fin de producir de nuevas } \\
\text { ideas para su posterior análisis } \\
\text { cada participante va diciendo } \\
\text { libremente lo que se le ocurre } \\
\text { sobre un tema o pregunta } \\
\text { específica sin valorar las ideas } \\
\text { que van surgiendo. }\end{array}$ \\
\hline 12 & $\begin{array}{l}\text { Prácticas } \\
\text { (laboratorio y } \\
\text { similares) }\end{array}$ & 6 & $\begin{array}{l}\text { Modalidad de enseñanza práctica } \\
\text { donde el contenido principal de } \\
\text { lo que será aprendido no se } \\
\text { proporciona por vía transmisivo- } \\
\text { receptiva, sino que es descubierto } \\
\text { por el discente antes de ser } \\
\text { asimilado en su estructura } \\
\text { cognitiva (Navaridas, 2004). }\end{array}$ & $\begin{array}{l}\text { Método de enseñanza práctico y } \\
\text { activo donde el contenido } \\
\text { principal de lo que será } \\
\text { aprendido es demostrado o } \\
\text { practicado por el alumno, a } \\
\text { partir de la guía del profesor y de } \\
\text { unos materiales concretos. }\end{array}$ \\
\hline
\end{tabular}




\begin{tabular}{|c|c|c|c|c|}
\hline & MÉTODO & $\begin{array}{l}\text { NÚMERO } \\
\text { DE } \\
\text { FUENTES }\end{array}$ & DEFINICIÓN ORIGINAL & DEFINICIÓN \\
\hline 13 & Trabajo de grupo & 4 & $\begin{array}{l}\text { El profesor programa diversas } \\
\text { actividades que deberán afrontar } \\
\text { los equipos de trabajo formados } \\
\text { por los estudiantes. Un buen } \\
\text { equipo de trabajo es aquél en que } \\
\text { se optimizan las capacidades de } \\
\text { todos los componentes } \\
\text { (Navaridas, 2004). }\end{array}$ & $\begin{array}{l}\text { Realización en equipos de } \\
\text { trabajo de actividades } \\
\text { programadas por el profesor que } \\
\text { habitualmente concluye con la } \\
\text { elaboración de un documento } \\
\text { para su evaluación. }\end{array}$ \\
\hline 14 & Investigación & 4 & $\begin{array}{l}\text { Método de enseñanza práctica } \\
\text { que requiere al estudiante } \\
\text { identificar el problema objeto de } \\
\text { estudio, formularlo con precisión, } \\
\text { desarrollar los procedimientos } \\
\text { pertinentes, interpretar los } \\
\text { resultados y sacar las conclusiones } \\
\text { oportunas del trabajo realizado } \\
\text { (Navaridas, 2004). }\end{array}$ & $\begin{array}{l}\text { Método de enseñanza que } \\
\text { reproduce las fases y } \\
\text { procedimientos de la } \\
\text { investigación científica. Requiere } \\
\text { al estudiante formular el } \\
\text { problema, desarrollar las } \\
\text { hipótesis y los procedimientos } \\
\text { pertinentes para contrastarlas, } \\
\text { interpretar los resultados y sacar } \\
\text { conclusiones. }\end{array}$ \\
\hline 15 & $\begin{array}{l}\text { Estudio } \\
\text { independiente }\end{array}$ & 4 & $\begin{array}{l}\text { El trabajo autónomo de los } \\
\text { estudiantes permite que cada uno } \\
\text { de ellos vaya siguiendo su propio } \\
\text { ritmo y acomodando el } \\
\text { aprendizaje a sus particulares } \\
\text { circunstancias (Zabalza, 2003). }\end{array}$ & $\begin{array}{l}\text { Trabajo autónomo de los } \\
\text { estudiantes vinculado a la } \\
\text { materia desarrollada en las } \\
\text { clases, que permite que cada uno } \\
\text { siga su propio ritmo } \\
\text { acomodando el aprendizaje a sus } \\
\text { particulares circunstancias. }\end{array}$ \\
\hline 16 & $\begin{array}{l}\text { Trabajos o } \\
\text { ensayos } \\
\text { (individuales) }\end{array}$ & 3 & $\begin{array}{l}\text { Trabajos que realiza el alumno. } \\
\text { Algunos ejemplos pueden ser: } \\
\text { recensiones, trabajos monográficos, } \\
\text { memorias o proyectos } \\
(U R V, 2006) \text {. }\end{array}$ & $\begin{array}{l}\text { Trabajos realizados por los } \\
\text { estudiantes individualmente, } \\
\text { tales como recensiones, } \\
\text { monográficos, memorias o } \\
\text { ensayos. }\end{array}$ \\
\hline 17 & $\begin{array}{l}\text { Aprendizaje } \\
\text { acción }\end{array}$ & 3 & $\begin{array}{l}\text { El aprendizaje acción se construye } \\
\text { sobre la relación entre reflexión y } \\
\text { acción. Formaliza el aprendizaje } \\
\text { reflexivo y legitimiza la asignación } \\
\text { de tiempo y espacio para ello, a } \\
\text { través de un grupo que trabaja a lo } \\
\text { largo de un amplio periodo de } \\
\text { tiempo (McGill y Brockbank, } \\
\text { 2004). }\end{array}$ & $\begin{array}{l}\text { Se lleva a cabo a través de } \\
\text { grupos que se reúnen } \\
\text { regularmente donde los } \\
\text { integrantes exponen proyectos, } \\
\text { problemas o dificultades que } \\
\text { encuentran en sus entornos de } \\
\text { trabajo. Se utiliza el conocimiento } \\
\text { y el apoyo del grupo para } \\
\text { elaborar soluciones o propuestas } \\
\text { de mejora. }\end{array}$ \\
\hline 18 & $\begin{array}{l}\text { Vídeos y otras } \\
\text { técnicas } \\
\text { audiovisuales }\end{array}$ & 3 & $\begin{array}{l}\text { Son técnicas que utilizan la } \\
\text { imagen y el sonido como } \\
\text { lenguajes de comunicación y } \\
\text { expresión (Navaridas, 2004). }\end{array}$ & $\begin{array}{l}\text { Utilización de la imagen y el } \\
\text { sonido como lenguajes de } \\
\text { comunicación. Su característica } \\
\text { diferencial es que el alumno } \\
\text { recibe la información en formato } \\
\text { multimedia. }\end{array}$ \\
\hline 19 & $\begin{array}{l}\text { Dinámicas de } \\
\text { grupo }\end{array}$ & 3 & $\begin{array}{l}\text { Conjunto de métodos prácticos } \\
\text { de trabajo con grupos. Técnicas } \\
\text { de trabajo basadas en la dinámica } \\
\text { del grupo (López-Yarto, 1997). }\end{array}$ & $\begin{array}{l}\text { Métodos prácticos y técnicas de } \\
\text { trabajo basados en la dinámica } \\
\text { grupal. }\end{array}$ \\
\hline
\end{tabular}




\begin{tabular}{|c|c|c|c|c|}
\hline & MÉTODO & $\begin{array}{l}\text { NÚMERO } \\
\text { DE } \\
\text { FUENTES }\end{array}$ & DEFINICIÓN ORIGINAL & DEFINICIÓN \\
\hline 20 & Exámenes & 3 & $\begin{array}{l}\text { Pruebas a desarrollar, pruebas de } \\
\text { preguntas cortas, pruebas objetivas } \\
\text { tipo test, pruebas prácticas o } \\
\text { pruebas orales (URV, 2006). }\end{array}$ & $\begin{array}{l}\text { Pruebas de evaluación en } \\
\text { diferentes formatos que pueden } \\
\text { incluir preguntas de diverso tipo, } \\
\text { pruebas tipo test, resolución de } \\
\text { problemas o pruebas orales. }\end{array}$ \\
\hline 21 & $\begin{array}{l}\text { Prácticas } \\
\text { profesionales }\end{array}$ & 2 & $\begin{array}{l}\text { Estadios de formación de la } \\
\text { titulación, en general en empresas } \\
\text { o instituciones del sector (URV, } \\
\text { 2006). }\end{array}$ & $\begin{array}{l}\text { Segmento de formación en el } \\
\text { cual el proceso de aprendizaje se } \\
\text { realiza en empresas o } \\
\text { instituciones del sector, } \\
\text { habitualmente bajo la guía de un } \\
\text { tutor. }\end{array}$ \\
\hline 22 & Presentaciones & 2 & $\begin{array}{l}\text { Exposición oral por parte de los } \\
\text { alumnos de un tema concreto o } \\
\text { de un trabajo (previa presentación } \\
\text { escrita) (URV, 2006). }\end{array}$ & $\begin{array}{l}\text { Exposición por parte de los } \\
\text { alumnos de un trabajo } \\
\text { previamente desarrollado, } \\
\text { normalmente apoyada por } \\
\text { recursos audiovisuales. }\end{array}$ \\
\hline 23 & $\begin{array}{l}\text { Mapas } \\
\text { conceptuales }\end{array}$ & 2 & $\begin{array}{l}\text { Consiste en la representación } \\
\text { gráfica de los conceptos } \\
\text { fundamentales de la materia de } \\
\text { aprendizaje y de sus relaciones } \\
\text { entre sí con el fin de ayudar a los } \\
\text { estudiantes a ver su significado } \\
\text { (Navaridas, 2004). }\end{array}$ & $\begin{array}{l}\text { Trabajo cuyo objetivo es la } \\
\text { representación gráfica de los } \\
\text { conceptos fundamentales de la } \\
\text { materia de aprendizaje así como } \\
\text { de las relaciones entre ellos. }\end{array}$ \\
\hline 24 & $\begin{array}{l}\text { Método de } \\
\text { dilemas morales }\end{array}$ & 2 & $\begin{array}{l}\text { Se trata de una situación } \\
\text { problemática, generalmente } \\
\text { presentada de forma oral, a través } \\
\text { de la cual los participantes } \\
\text { deberán escoger forzosamente } \\
\text { una alternativa, que puede ser } \\
\text { previamente razonada y } \\
\text { contrastada en un debate. Su } \\
\text { fundamentación está muy ligada } \\
\text { a la clarificación de valores, } \\
\text { ejercicios de toma de decisiones } \\
\text { y a los estudios de casos (Jares, } \\
2002 \text { ). }\end{array}$ & $\begin{array}{l}\text { Se trata de una situación } \\
\text { problemática desde el punto de } \\
\text { vista moral en la que los } \\
\text { participantes deben escoger una } \\
\text { alternativa que normalmente es } \\
\text { previamente razonada y } \\
\text { contrastada en un debate. }\end{array}$ \\
\hline 25 & $\begin{array}{l}\text { Ejercicio de } \\
\text { clarificación de } \\
\text { valores }\end{array}$ & 2 & $\begin{array}{l}\text { Resulta útil como vía para tomar } \\
\text { consciencia de los códigos de } \\
\text { valoración que tenemos, } \\
\text { sensibilizar sobre el sistema de } \\
\text { relaciones en el aula y centro y } \\
\text { facilitar su construcción desde } \\
\text { una óptica de respeto y mutua } \\
\text { ayuda, así como sobre diferentes } \\
\text { tipos de contenido (procesos de } \\
\text { discriminación, violencia, } \\
\text { armamentismo, derechos } \\
\text { humanos, desarrollo, etc.) } \\
\text { (Jares, 2002). }\end{array}$ & $\begin{array}{l}\text { Tarea que tiene por objeto } \\
\text { fundamental tomar conciencia de } \\
\text { los códigos de valoración de } \\
\text { cada participante. }\end{array}$ \\
\hline
\end{tabular}

Fuente: elaboración propia. 


\section{CONCLUSIONES Y PRÓXIMOS PASOS}

En 1980 ya se hablaba de una "acción múltiple que hoy exige la crisis universitaria y la de las enseñanzas medias: la renovación de los métodos de enseñanza" (Román, 1980, p.30). Más de veinticinco años después se recogía: "el diagnóstico de la situación actual podría sintetizarse afirmando que la reforma de las metodologías educativas se percibe como un proceso que es imprescindible abordar para una actualización de la oferta formativa de las universidades españolas." (MEC, 2006, p. 7).

Resulta significativo que después de tan largo periodo de tiempo siga manifestándose la necesidad de reflexionar sobre la metodología, como un eco que señala de forma recursiva esta asignatura pendiente de la profesión docente. Puede deberse al hecho de que no se hayan percibido avances significativos en este terreno, o que los avances que se han logrado no resulten suficientes. En cualquiera de los dos casos es un hecho cierto que, por ser la metodología una de las claves de la función docente, sigue siendo necesario reflexionar sobre su conocimiento profundo y su aplicación práctica. Sin embargo, como hemos recogido líneas arriba, la investigación sobre los métodos de enseñanza es un terreno lleno de dificultades terminológicas y taxonómicas.

Por eso no siempre resulta sencillo para el profesorado (sobre todo para aquel que no tiene formación específica en docencia) escoger y aplicar los distintos métodos de enseñanza que su labor docente requiere. En este sentido será poco todo el esfuerzo que se haga para clarificar qué es un método de enseñanza y cómo debe aplicarse, y ello no solo para la práctica de aula, sino también para el diseño de programas de formación del profesorado y para la estructuración de instrumentos de recogida de información que la investigación requiere.

El campo de estudio en torno a los métodos de enseñanza resulta francamente amplio, y sigue siendo preciso dedicar esfuerzos investigadores a avanzar en este terreno. En este trabajo hemos creado un listado de veinticinco métodos de enseñanza con sus definiciones, basándonos en veintidós fuentes documentales y en un panel de nueve expertos, y hemos expuesto algunas precisiones terminológicas que bajo nuestra perspectiva resultan necesarias.

El siguiente paso consiste en clasificar los métodos de enseñanza que hemos incluido en el inventario. Desde nuestro punto de vista esta clasificación debería tener al menos dos ejes:

- Por un lado, deberíamos poder clasificar los métodos de enseñanza en función de la finalidad (o finalidades) educativas a las que cada uno de ellos sirve. De este modo, los docentes podrían disponer de un esquema básico para relacionar las actividades de aprendizaje con las competencias que persiguen desarrollar en sus estudiantes.

- Por otro lado, sería clarificador poder conectar los métodos de enseñanza con las técnicas que contienen. Esto ayudaría de forma importante a definir la articulación interna de cada método, además de apoyar significativamente los programas de formación de docentes.

Bajo nuestra perspectiva, el modelo planteado por el Espacio Europeo de Educación Superior, que ha centrado muchos de los esfuerzos investigadores en el estudio 
de las competencias, trae también a un primer plano la investigación sobre la metodología docente, y pone de manifiesto que la ciencia de los métodos de enseñanza es más actual que nunca.

\section{REFERENCIAS BIBLIOGRÁFICAS}

ALCOBA, J. (2010). Los métodos de enseñanza en la estrategia docente de las Instituciones de Educación Superior. Un estudio sobre Escuelas de Negocios. Sevilla: Universidad Pablo de Olavide.

AMAT, O. (2002). Aprender a enseñar. Una visión práctica de la formación de formadores. Barcelona: Gestión 2000.

ANDREU, M. A. et. al. (2005). Estrategias de enseñanza-aprendizaje en la Universidad Politécnica de Valencia. Disponible en: http://www.upct.es/ dcta/Conver gencia/Otros_documentos/Estrategias_de_ensenanza_en_la_UPV.pdf.

BOURNER, T. (1997). Teaching methods for learning outcomes. Education + Training, 39(9), 344-348.

BUSINESSWEEK (2006). 2006 Full-Time MBA Profiles. Disponible en: http://www. businessweek.com/bschools/06/geographic.htm. [Consultado: 03-08, 2007].

DOMÍNGUEZ, G. (Coord.); AMADOR, L., HERMOSILLA, J. y LOZANO, L. (2007). Diseño de proyectos y procesos de formación: Niveles y modalidades. Aspectos didácticos o curriculares (docentes). En Materiales del curso en Gestión de Calidad de la Formación. Madrid: UNED-INAP.

EUROFM (2007). European FM Education Guide. Naarden: EuroFM.

FERNÁNDEZ MARCH, A. (2006). Metodologías activas para la formación de competencias. Educatio Siglo XXI, 24, 35-56.

FERNÁNDEZ PÉREZ, M. (1994). Las tareas de la profesión de enseñar. Práctica de la racionalidad curricular. Didáctica aplicable. Madrid: Siglo XXI.

FERNÁNDEZ SERRAT, M. L. (2007). Planificación del aprendizaje. En M. C. Fonseca Mora y J. I. Aguaded Gómez (Dir.), Enseñar en la Universidad. Experiencias y propuestas para la docencia universitaria. La Coruña: Netbiblo.

FERRÁNDEZ, A. y SARRAMONA, J. (1975). La Educación: constantes y problemática actual. Barcelona: CEAC.

GAIRÍN, J. (2005). Nociones de desarrollo curricular. El crédito europeo. En C. Chamorro y P. Sánchez (Eds.), Iniciación a la docencia universitaria. Manual de ayuda. Madrid: Universidad Complutense de Madrid-ICE.

HONOLULU COMMUNITY COLLEGE, FACULTY DEVELOPMENT (s.f.). Common Teaching Methods. Disponible en: http://honolulu.hawaii.edu/intranet/committees/ FacDevCom/guidebk/teachtip/comteach.htm. [Consultado: 03-08, 2007].

JARES, X. (2002). Métodos y actividades didácticas. En M. Rodriguez Rojo (Ed.), Didáctica General. Qué y cómo enseñar en la sociedad de la información. Madrid: Biblioteca nueva.

KOLB, D. A. (1984). Experiential Learning. Experience as the source of learning and development. New Jersey: Prentice Hall.

LÓPEZ CAMPS, J. (2005). Planificar la formación con calidad. Madrid: CissPraxis. 
LÓPEZ-YARTO, L. (1997). Dinámica de grupos. Cincuenta años después. Bilbao: Desclée de Brouwer.

MARTÍN-MOLERO, F. (1993). El método: su teoría y su práctica. Madrid: Dykinson.

MARZANO, R. J. (1998). A Theory-Based Meta-Analysis of Research on Instruction. Denver, Co.: McREL. Disponible en: http://www.mcrel.org/PDF/Instruction/ 5982RR_InstructionMeta_Analysis.pdf. [Consultado: 03-02, 2008].

MCGILL, I. y BROCKBANK, A. (2004). The action learning handbook. Londres: Routledge Falmer.

MEC (2006). Propuestas para la renovación de las metodologías educativas en la universidad. Madrid: Secretaría de Estado de Universidades e Investigación. Dirección General de Universidades. MEC.

MONTERREY, INSTITUTO TECNOLÓGICO Y DE ESTUDIOS SUPERIORES DE. (2006). Técnicas didácticas. Disponible en: http://www.sistema.itesm.mx/va/dide/tecni cas_didacticas/otrastecnicas.htm. [Consultado: 03-08, 2007].

NAVARIDAS NALDA, F. (2004). Estrategias didácticas en el aula universitaria. Logroño: Universidad de la Rioja. Servicio de Publicaciones.

RAE (2008). Diccionario de la Lengua Española (22ํe․) Disponible en: http://bus con.rae.es/drael/. [Consultado: 09-09, 2008].

ROMÁN, J. M. (1980). Introducción a los métodos activos de enseñanza. En J. M. Román (Ed.), Métodos activos para enseñanzas medias y universitarias. Madrid: Cincel.

UNIVERSITAT ROVIRA I VIRGILI (2006). Guia de Metodologies Docents (2006). Tarragona: Universitat Rovira i Virgili.

ZABALZA, M. A. (2003). Competencias docentes del profesorado universitario. Madrid: Narcea. 